\title{
Estimación de distribuciones diamétricas para Pinus patula con la función Weibull
}

\section{Estimation of diameter distributions for Pinus patula with the Weibull function}

\author{
Eloísa Pérez-López', Wenceslao Santiago-García²*, Gerónimo Quiñonez-Barraza³, \\ Gerardo Rodríguez-Ortiz ${ }^{4}$ Elías Santiago-García y y Faustino Ruiz-Aquino²
}

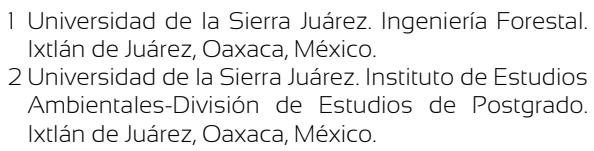

2 Universidad de la Sierra Juárez. Instituto de Estudios Ambientales-División de Estudios de Postgrado. Ixtlán de Juárez, Oaxaca, México.

\author{
3 Instituto Nacional de Investigaciones Forestales, \\ Agrícolas y Pecuarias. Centro de Investigación \\ Regional Norte Centro. Campo Experimental Valle \\ del Guadiana. Durango, Durango, México. \\ 4 Instituto Tecnológico del Valle de Oaxaca. División \\ de Estudios de Posgrado e Investigación. Oaxaca, \\ México
}

5 Dirección Técnica Forestal de la Comunidad de Ixtlán de Juárez. Oaxaca, México.

* Autor de correspondencia.wsantiago@unsij.edu.mx

\section{RESUMEN}

La predicción implícita es una herramienta valiosa para conocer el tipo de productos que se pueden obtener del bosque en un tiempo determinado y, de esta forma, planificar el manejo forestal sostenible. En la Sierra Norte de Oaxaca, México, existen rodales coetáneos de Pinuspatula, una especie maderable de rápido crecimiento e importancia económica y ecológica. El objetivo de este estudio fue estimar el crecimiento y rendimiento maderable de esta especie a través de dos sistemas de predicción con el enfoque de modelos de distribución en clases diamétricas. La base de datos consistió en 132 distribuciones diamétricas, provenientes de 66 sitios permanentes de muestreo de $400 \mathrm{~m}^{2}$ localizados en Ixtlán de Juárez, Oaxaca, México. La distribución diamétrica del rodal se estimó mediante la función de densidad de probabilidades Weibull con los métodos de predicción de parámetros y predicción de percentiles. Asimismo, se ajustaron modelos de proyección del diámetro mínimo y diámetro medio cuadrático del rodal para recuperar los parámetros y estimar la estructura diamétrica de rodales a medida que cambia la edad. El mejor método fue el de predicción de parámetros, debido al sesgo menor en la predicción del volumen total con respecto a un sistema de proyección explícito diseñado para la especie. Por tanto, es preferente el uso de este sistema de predicción implícita para planificar el manejo de los rodales coetáneos de $P$. patula.

PALABRAS CLAVE: método de momentos, predicción de parámetros, predicción de percentiles, predicción implícita, prueba de Kolmogorov-Smirnov, regresión.

\section{ABSTRACT}

The implicit prediction is an important tool to know the type of products that can be obtained from the forest in a given time, and thus to plan the sustainable forest management. In the Sierra Norte de Oaxaca, Mexico, there are even-aged stands of Pinus patula, a fastgrowing timber species with economic and ecological value. The aim of this study was to estimate the growth and yield timber of this species with the diameter distribution models approach. The database consisted of 132 diameter distributions from 66 permanent sampling plots of $400 \mathrm{~m}^{2}$, located in Ixtlán de Juárez, Oaxaca, Mexico. Stand diameter distribution was estimated through Weibull function with parameters prediction and percentile prediction approaches. Also, minimum diameter and quadratic mean diameter projection models were fitted to recover the parameters and to estimate the diameter structure of stands on the future. The best system was the prediction of parameters because of this had the smaller bias in the prediction of the total volume with respect to an explicit projection system designed for the species. Therefore, it is recommended to use this implicit prediction system to plan the management of even-aged stands of P. patula.

KEYWORDS: moments methodology, parameter prediction, percentiles prediction, implicit prediction, Kolmogorov-Smirnov test, regression. 


\section{INTRODUCCIÓN}

En el manejo forestal se requiere el uso y diseño de herramientas cuantitativas para la toma de decisiones, los modelos de crecimiento y rendimiento forestal son los instrumentos más utilizados para lograr tal fin. Estos modelos tienen como función determinar la evolución en el tiempo de una o varias variables dendrométricas (del árbol individual o de rodal) que se definen en el sistema a estudiar. Los modelos a nivel de rodal son los más adecuados para la planeación del manejo de rodales coetáneos. Sin embargo, en algunos casos se requiere información más detallada para la toma de decisiones. Los modelos de distribución diamétrica permiten realizar una desagregación del volumen total para conocer la distribución de productos maderables, en tanto que, los modelos de árbol individual permiten estimar variables de crecimiento a ese nivel, así como conocer el número de árboles de cada clase diamétrica (Quevedo, Moret y Jerez, 2003; Gómez-García, Crecente-Campo, Pérez-Rodríguez y Diéguez-Aranda, 2013; Hirigoyen y Rachid, 2014).

La estimación de las distribuciones diamétricas se realiza a través de funciones de densidad de probabilidades (fdp) (Hafley y Schreuder, 1977), siendo las distribuciones Beta, Johnson $S_{B}$, Weibull, lognormal, gamma y normal las comúnmente usadas para describir distribuciones diamétricas de rodales en bosques coetáneos e incoetáneos (Bailey y Dell, 1973; Hafley y Schreuder, 1977; Maltamo, Puumalainen y Päivinen, 1995).

La función Weibull derivada por Waloddi Weibull (1939) en estudios de resistencia de materiales, ha sido la más utilizada en la ciencia forestal por su relativa facilidad de aplicación, y fue introducida por Bailey y Dell (1973) para modelar distribuciones diamétricas de Pinus taeda, P. echinata, P. banksiana y Pseudotsuga menziesii. Entre las ventajas de la fdp Weibull están su manejo matemático sencillo y la flexibilidad para adoptar diferentes formas, desde la "J" invertida hasta distribuciones en forma de campana, con diferentes grados de sesgo; además, se puede integrar analíticamente para generar una función de distribución acumulativa de forma cerrada (Bailey y Dell, 1973; Maldonado y Navár, 2002) para determinar el número de árboles entre una clase diamétrica inferior y una superior. Esta función puede caracterizarse de manera dinámica a partir de la predicción de los parámetros, esto es, después de estimar los parámetros de localización, escala y forma ( $a$, $b$ y $c$, respectivamente) con alguno de los métodos diseñados para ello (Torres-Rojo, Magaña-Torres y AcostaMireles, 2000), se pueden relacionar con variables del rodal usando modelos lineales o no lineales. Con este método, se han obtenido resultados de ajuste estadísticamente adecuados que además permiten obtener directamente los parámetros de las distribuciones futuras proyectadas y facilita la planeación de los regímenes de cortas intermedias (Reynolds, Burk y Huang, 1988; García, Cañadas y Montero, 2002). Otra técnica para estimar los parámetros de la función Weibull es la mezcla entre métodos de estimación de parámetros y percentiles; como el método de recuperación de parámetros que consiste en recobrar los parámetros de la distribución a partir de sus momentos nocentrales y momentos centrales, al tener como base un conjunto de percentiles los cuales son fáciles de modelar de manera dinámica (Hyink y Moser, 1983).

En México se han generado modelos basados en la fdp Weibull para estimar distribuciones diamétricas en rodales coetáneos e incoetáneos y plantaciones de coníferas, entre las que destacan Pinus caribaea var. hondurensis Barr y Golf., P. durangensis Martínez, P. cooperi Blanco, P. engelmannii Carr., P. arizonica Engl. y P. patula (Montero y Fierros, 2000; Maldonado y Návar, 2002; Magaña, Torres, Rodríguez, Aguirre y Fierros, 2008; Santiago-García et al., 2014; Quiñonez, De los Santos, Cruz, Velázquez y Ramírez, 2015).

\section{OBJETIVOS}

Predecir el rendimiento maderable por clase diamétrica para Pinus patula Schiede ex Schlechtendal \& Chamisso con el uso de la función de densidad de probabilidades Weibull y las técnicas de predicción de parámetros y predicción de percentiles. 


\section{MATERIALES Y MÉTODOS}

\section{Área de estudio}

El estudio se llevó a cabo en rodales coetáneos de Pinus patula en bosques del predio de Ixtlán de Juárez, Oaxaca, México (Fig. 1), entre las coordenadas 17018'16" y $17^{\circ} 30^{\prime} 00^{\prime \prime}$ latitud norte y $96^{\circ} 31^{\prime} 38^{\prime \prime}$ y $96^{\circ} 22^{\prime} 00^{\prime \prime}$ longitud oeste, con una altitud media de $2780 \mathrm{~m}$ snm. La región se localiza en la provincia fisiográfica denominada Sistema
Montañoso del Norte de Oaxaca. Dicha provincia abarca la mitad septentrional de Oaxaca y áreas adyacentes de Puebla y Veracruz. Los tipos de climas predominantes en la zona son templado subhúmedo y templado subhúmedo con lluvias en verano, con temperatura media anual que va de $14^{\circ} \mathrm{C}$ a $18^{\circ} \mathrm{C}$. Los grupos de suelo más comunes en el área corresponden a acrisol, luvisol y cambisol. El principal tipo de vegetación presente corresponde a bosques de pinoencino (Servicios técnicos forestales de Ixtlán de Juárez [STF], 2015).

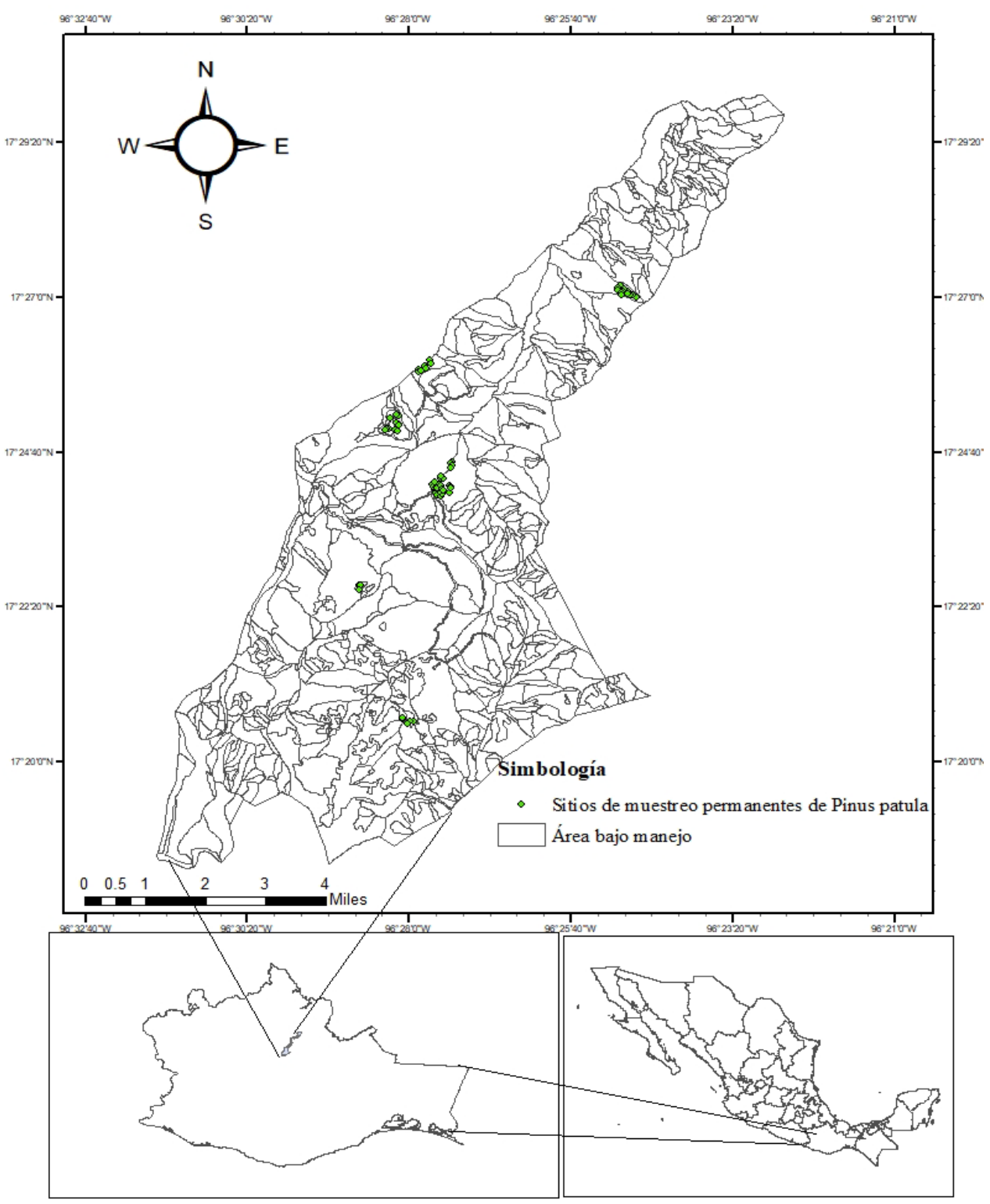

Figura 1. Localización del área de estudio. 


\section{Datos dasométricos}

Para el análisis se utilizó una base de datos de 132 distribuciones diamétricas, provenientes de dos mediciones realizadas en 66 sitios permanentes de investigación silvícola de forma cuadrada $\left(400 \mathrm{~m}^{2}\right)$ en rodales coetáneos de Pinus patula, los sitios se establecieron en 2015 cubriendo diferentes intervalos de densidad, calidad de sitio (20 m - 38 m) y edad (de 5 años a 80 años), vueltos a medir en 2016. Dentro de cada sitio se midió el diámetro normal $(D n)$ de todos los árboles, la altura $(A t)$ de al menos ocho árboles por sitio, de los cuales, cuatro se identificaron como dominantes de acuerdo con la definición de altura dominante que corresponde a los 100 árboles de mayor altura y diámetro por hectárea (Assmann, 1970; Alder, 1980).

Las variables de estado del rodal fueron: altura total promedio $(A t, \mathrm{~m})$, altura dominante $(H d, \mathrm{~m})$, área basal $(A b$, $\left.\mathrm{m}^{2} \mathrm{ha}^{-1}\right)$, número de árboles $\left(\mathrm{Na} \mathrm{ha}^{-1}\right)$, diámetro promedio $(D m, \mathrm{~cm})$, diámetro medio cuadrático $(D q, \mathrm{~cm})$, diámetro mínimo $(D$ min, $\mathrm{cm})$ y volumen $\left(V, \mathrm{~m}^{3} \mathrm{ha}^{-1}\right)$. El diámetro medio cuadrático corresponde al diámetro del árbol de área basal media:

$$
D q=\sqrt{\frac{40000}{\pi} \times \frac{A b}{N a}}
$$

La altura total para los individuos no medidos fue obtenida con el modelo altura-diámetro ajustado por López-Villegas et al. (2017):

$$
\begin{aligned}
A t=46.06167 & \times[1 \\
& -\exp (-0.023647 \times D n)]^{0.936185}
\end{aligned}
$$

El volumen del fuste total con corteza para cada árbol $(v$, $\mathrm{m}^{3}$ ) se calculó con la ecuación de doble entrada ajustada por Rodríguez-Justino (2017):

$$
v=0.000074 \times D n^{1.610374} \times A t^{1.213333}
$$

En la tabla 1 se presentan las estadísticas descriptivas de los datos empleados en el ajuste de los sistemas de predicción implícita.

TABLA 1. Resumen estadístico de los datos utilizados en el ajuste para estimar los parámetros de la función Weibull.

\begin{tabular}{cccccc}
\hline Variable & Observaciones & Media & Desviación estándar & Mínimo & Máximo \\
\hline$A t$ & & 9.57 & 4.87 & 1.88 & 36.74 \\
$D n$ & 8015 & 11.88 & 6.96 & 2.10 & 58.30 \\
$E^{\dagger}$ & & 14.81 & 11.54 & 5.00 & 80.00 \\
$H d$ & 15.39 & 6.27 & 4.55 & 35.41 \\
$A b$ & 22.59 & 13.74 & 1.35 & 68.26 \\
$N a$ & 1517.99 & 1011.85 & 300.00 & 6050.00 \\
$D m$ & 13.93 & 6.49 & 5.80 & 39.84 \\
$D q$ & 132 & 7.16 & 5.87 & 43.52 \\
$D$ min & & 2.73 & 2.87 & 17.00 \\
$V$ & & 180.66 & 165.86 & 3.93 & 681.11 \\
$E^{5}$ & & 18.72 & 5.00 & 75.25 \\
\hline
\end{tabular}

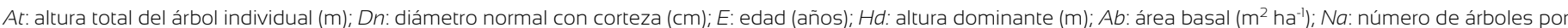

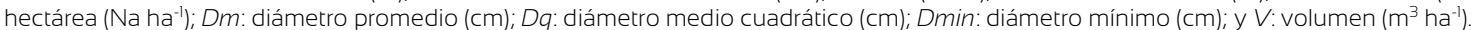

†Edad del árbol individual.

${ }^{5}$ Edad promedio de la distribución diamétrica 
La calidad del sitio forestal (índice de sitio, IS) y su correspondiente altura dominante se estimaron mediante el modelo polimórfico de Levakovic II, a una edad base de 40 años (Pérez-López et al., 2017; Santiago-García et al., 2017):

$$
I S=52.76080 \cdot\left[\frac{40}{E \cdot\left[-1+(H d / 52.76080)^{-0.32193}\right]+40}\right]^{3.10631}
$$

$$
H d=52.76080 \cdot\left[\frac{E}{40 \cdot\left[-1+(I S / 52.76080)^{-0.32193}\right]+E}\right]^{3.10631}
$$

\section{Función de distribución Weibull}

La distribución Weibull triparamétrica está definida por la función de densidad probabilística siguiente (Bailey y Dell, 1973; Clutter, Forston, Pienaar, Brister y Bailey, 1983; Cao, 2004):

$$
\begin{gathered}
f(x)=\frac{c}{b} \cdot\left(\frac{x-a}{b}\right)^{c-1} \cdot \exp \left[-\left(\frac{x-a}{b}\right)^{c}\right] \\
(a \leq x \leq \infty)
\end{gathered}
$$

donde $f(x)$ es la probabilidad asociada con cada posible valor de la variable aleatoria $x$ (diámetro normal); $a, b$ y $c$ son los parámetros de localización, escala y forma, respectivamente.

La distribución por categoría diamétrica se determinó con la distribución acumulada de forma cerrada (Bailey y Dell, 1973; Clutter, Forston, Pienaar, Brister y Bailey, 1983):

$$
\begin{gathered}
f(x)=1-\exp \left[-\left(\frac{x-a}{b}\right)^{c}\right] \\
(a \leq x \leq \infty)
\end{gathered}
$$

La porción de la población comprendida dentro de un intervalo determinado está dada por la ecuación (Clutter, Forston, Pienaar, Brister y Bailey, 1983; Rennolls, Geary y Rollinson, 1985):

$P(L<X<U)=\exp \left[-\left(\frac{L-a}{b}\right)^{c}\right]-\exp \left[-\left(\frac{U-a}{b}\right)^{c}\right]$ donde $P$ es la proporción de árboles en la categoría diamétrica correspondiente, $L$ y $U$ son los límites inferior y superior de la categoría diamétrica, respectivamente, $X$ es la categoría diamétrica y exp indica la función exponencial; el resto se definió previamente.

El producto de la proporción de árboles $(P)$ en cada categoría diamétrica $(X)$ y la densidad del rodal determinan el número de árboles por clase diamétrica, es decir, cuántos árboles de cierto diámetro se tendrán en una edad determinada (Quiñonez, De los Santos, Cruz, Velázquez y Ramírez, 2015).

\section{Predicción de parámetros de la fdp Weibull}

Los parámetros de la fdp Weibull se estimaron de acuerdo con la distribución diamétrica observada en cada sitio de muestreo; estos valores se obtuvieron mediante el procedimiento CAPABILITY de SAS/ETS® 9.3, el cual utiliza métodos basados en máxima verosimilitud para la estimación de parámetros (SAS Institute Inc., 2011).

Una vez estimados los parámetros fueron relacionados con los atributos dasométricos del rodal: área basal $(A b)$, diámetro mínimo (Dmin), diámetro promedio $(D m)$, diámetro medio cuadrático $(D q)$, número de árboles $(\mathrm{Na})$, edad $(E)$, altura dominante $(H d)$ e índice de sitio $(I S)$ por medio de regresión lineal, con el procedimiento STEPWISE (selección por pasos) de SAS (SAS Institute Inc., 2011) (Maldonado y Návar, 2002). En este caso se consideraron dos ecuaciones para cada parámetro (Tabla 2).

El coeficiente de asimetría $(C a)$ se calculó con la expresión:

$$
C_{a}=\frac{n}{(n-1) \cdot(n-2)} \sum\left(\frac{y_{i}-\bar{y}}{s}\right)^{3}
$$

donde:

$n:$ número de observaciones

$s$ es la desviación estándar

$y_{i}$ : valor observado de la distribución diamétrica

$\bar{y}$ : valor promedio de la distribución diamétrica 
TABLA 2. Ecuaciones ajustadas para la predicción de los parámetros de la función Weibull.

\begin{tabular}{crrr}
\hline Parámetro & \multicolumn{1}{c}{ Ecuación } & & Clave \\
\hline Localización (a) & $a$ & $=\beta_{0} \cdot D \min$ & a.E1 \\
& $a=\beta_{0}+\beta_{1} \cdot b+\beta_{2} \cdot D m+\beta_{3} \cdot D q$ & a.E2 \\
& $b=\beta_{0}+\beta_{1} \cdot c+\beta_{2} \cdot \ln (D \min )^{2}+\beta_{3} \cdot D q$ & b.E1 \\
Escala (b) & $b=\beta_{0}+\beta_{1} \cdot c+\beta_{2} \cdot D \min ^{2}+\beta_{3} \cdot D q$ & b.E2 \\
& $c=\beta_{0} \cdot D q+\beta_{1} \cdot D m+\beta_{2} \cdot I S$ & c.E1 \\
Forma (c) & $c=\beta_{0} \cdot D q+\beta_{1} \cdot D m+\beta_{2} \cdot I S+\beta_{3} \cdot C a$ & c.E2
\end{tabular}

Dmin: diámetro mínimo (cm); Dm: diámetro promedio (cm); Dq: diámetro medio cuadrático; IS: índice de sitio; Ca: coeficiente de asimetría de la distribución diamétrica; In: logaritmo natural y ßi: parámetros a estimar.

\section{Predicción de percentiles}

Para este método se requieren al menos dos percentiles de la distribución diamétrica del rodal; los cuales se ajustaron como funciones del diámetro medio cuadrático del rodal $(D q)$, en este estudio los percentiles 55 y 85 resultaron adecuados. Así, las ecuaciones de predicción de percentiles tomaron la forma siguiente:

$$
\begin{aligned}
& p 55=\alpha \cdot D q^{\beta} \\
& p 85=\alpha \cdot D q^{\beta}
\end{aligned}
$$

donde:

p55: percentil 55 de la distribución diamétrica del rodal p85 : percentil 85 de la distribución diamétrica del rodal $D q$ : diámetro medio cuadrático del rodal a y $\beta$ : parámetros por estimar

Los parámetros de la distribución Weibull fueron recuperados por el método de momentos, considerando el criterio de Pienaar y Rheney (1993). Las ecuaciones utilizadas se muestran a continuación:

parámetro de localización (a):

$$
a=D \min
$$

si $D \min <5 \mathrm{~cm}$, entonces

$$
a=\frac{D \min }{2}
$$

A partir del parámetro de localización fue posible estimar el parámetro de forma $(c)$ y de escala $(b)$ respectivamente:

$$
\begin{gathered}
c=\frac{\ln [-\ln (1-0.85) /-\ln (1-0.55)]}{\ln [(p 85-a) /(p 55-a)]} \\
b=-a \cdot \frac{\Gamma_{1}}{\Gamma_{2}}+\sqrt{\left(\frac{a}{\Gamma_{2}}\right)^{2} \cdot\left(\Gamma_{1}^{2}-\Gamma_{2}\right)+\frac{D_{q}^{2}}{\Gamma_{2}}}
\end{gathered}
$$

donde:

$$
\begin{aligned}
& \Gamma_{1}=\Gamma \cdot\left(1+\frac{1}{c}\right) \\
& \Gamma_{2}=\Gamma \cdot\left(1+\frac{2}{c}\right)
\end{aligned}
$$

$\Gamma($.$) : función Gamma$

Dmin : diámetro mínimo del rodal $(\mathrm{cm})$

\section{Estimación del número de árboles}

Para la predicción del rendimiento maderable con distribuciones diamétricas fue necesario realizar la estimación del número de árboles sobrevivientes por hectárea a través del tiempo, esta variable fue estimada con la ecuación de mortalidad siguiente (Santiago-García et al., 2017): 


$$
N a_{2}=N a_{1} \cdot \exp \left[-0.01232\left(E_{2}-E_{1}\right)\right]
$$

donde $N a_{2}$ es el número de árboles vivos en la edad de proyección $E_{2}, N a_{1}$ corresponde al número de árboles en la edad inicial $E_{1}$.

\section{Modelo de proyección del diámetro mínimo y diámetro medio cuadrático}

El diámetro mínimo del rodal (percentil cero “ $p 0$ ”) es una variable de estado indispensable para realizar estimaciones de distribuciones diamétricas con el sistema basado en la fdp Weibull, debido a la estrecha relación que presenta con el parámetro de localización (a) (Santiago-García et al., 2014; Quiñonez, De los Santos, Cruz, Velázquez y Ramírez, 2015). Para la proyección del diámetro mínimo se utilizó la expresión anamórfica basada en el modelo de Chapman Richards (Amaro, Reed, Tomé y Temido, 1998):

$$
D \min _{2}=D \min _{1} \cdot\left[\frac{1-\exp \left(-\beta_{1} \cdot E_{2}\right)}{1-\exp \left(-\beta_{1} \cdot E_{1}\right)}\right]^{\beta_{2}}
$$

donde:

$D$ min 2 : diámetro mínimo del rodal en $E_{2}$

$D$ min 1 : diámetro mínimo del rodal en $E_{1}$

$E_{1}: \quad$ edad inicial del rodal (años)

$E_{2}: \quad$ edad de proyección del rodal (años)

$\beta_{i}: \quad$ parámetros por estimar

La proyección del diámetro medio cuadrático $(D q)$ del rodal se realizó mediante el ajuste del modelo compatible propuesto por Santiago-García et al. (2014) para predecir y proyectar el crecimiento de esta variable en el tiempo:

$$
\begin{gathered}
D q_{1}=\exp \left[\beta_{0}+\frac{\beta_{1}}{E_{1}} \cdot N a_{1}+\beta_{2} \cdot H d_{1}\right] \\
D q_{2}=D q_{1} \cdot \exp \left[\beta_{1} \cdot\left(\frac{N a_{2}}{E_{2}}-\frac{N a_{1}}{E_{1}}\right)+\beta_{2}\right. \\
\left.\cdot\left(H d_{2}-H d_{1}\right)\right]
\end{gathered}
$$

donde:

$D q_{1}$ : diámetro medio cuadrático del rodal en la edad inicial $E_{1}$ $D q_{2}$ : diámetro medio cuadrático del rodal a la edad de proyección $E_{2}$

$N a_{1}$ : número de árboles por hectárea en $E_{1}$

$N a_{2}$ : número de árboles por hectárea en $E_{2}$

$H d_{1}$ : altura dominante en $E_{1}$

$H d_{2}$ : altura dominante en $E_{2}$

$\beta_{i}: \quad$ parámetros por estimar

\section{Ajuste de los sistemas e indicadores estadísticos}

A través del método de mínimos cuadrados ordinarios, se ajustaron los modelos de predicción de parámetros, en tanto que, el modelo de proyección del diámetro mínimo y las ecuaciones de predicción de los percentiles 55 y 85 de la distribución diamétrica, se ajustaron a través de métodos iterativos adecuados para modelos de regresión no lineal, en este caso, se empleó el método de Gauss-Newton. Por su parte, el modelo compatible de crecimiento para diámetro medio cuadrático fue ajustado a través de regresión aparentemente no relacionada (SUR, por sus siglas en inglés), con el paquete SAS/ETS $®$, mediante el procedimiento MODEL (SAS Institute Inc., 2011).

La técnica de estimación de parámetros SUR produce estimadores consistentes y eficientes en presencia de correlaciones contemporáneas en un sistema de ecuaciones, además, permite compatibilidad total, de forma que los parámetros comunes de las ecuaciones toman los mismos valores mientras se cumpla con el criterio de minimización de cuadrados de los residuos (Borders, Souter, Bailey y Ware, 1987; Borders y Patterson, 1990; Galán, De los Santos y Valdez, 2008).

La bondad de ajuste de los modelos estudiados se midió a través del análisis numérico con la obtención de la suma de cuadrados del error ( $S C E$ ), la raíz del error cuadrático medio (RECM), el coeficiente de determinación $\left(R^{2}\right)$, el coeficiente de determinación ajustado por el número de parámetros $\left(\mathrm{R}^{2} a\right.$ atj), el sesgo promedio absoluto $(\bar{E})$ y el criterio de información de Akaike (AIC). 


\section{Bondad de ajuste de la fdp Weibull}

Para verificar la hipótesis de que las distribuciones diamétricas de las parcelas siguen una distribución Weibull, se utilizó el estadístico de Kolmogorov-Smirnov (KS), este se basa en las diferencias absolutas entre las distribuciones estimadas y observadas, para esto existen valores tabulados que permiten decidir si la diferencia máxima entre las distribuciones es significativa (Sokal y Rohlf, 1979). El cálculo de la diferencia máxima se realizó a través de la expresión siguiente:

$$
D \max |F(x)-S(x)|
$$

donde:

$D_{\text {max }}$ diferencia máxima entre las distribuciones acumuladas $F(x)$ : distribución acumulada teórica

$S(x)$ : distribución acumulada observada

Esto implica que dicha prueba solo es válida si los parámetros de la fdp Weibull son conocidos para cada distribución diamétrica (Torres-Rojo, Acosta y Magaña, 1992). Para esta prueba se utilizaron distintos niveles de significancia ( $a=0.05, a=0.10$ y $a=0.20$ ) para contrastar el número de parcelas no ajustadas a la fdp Weibull de acuerdo con cada criterio.

\section{Resultados}

\section{Predicción de parámetros de la fdp Weibull}

En la tabla 3 se presentan los resultados obtenidos de la prueba KS con diferentes niveles de significancia. De esta manera, se logró conocer la bondad de ajuste de la fdp Weibull a las estructuras diamétricas de los rodales de Pinus patula estudiados.

Pece, G. de Benítez y J. de Galíndez (2000) indican que el nivel de $a=0.20$ es el más exigente, porque reduce las desviaciones mínimas permitidas para el no rechazo de la concordancia. Con este nivel de significancia se trabajaron las 111 distribuciones diamétricas restantes y se procedió al ajuste de las ecuaciones de predicción de los parámetros de la fdp Weibull en relación con las variables de estado del rodal.

El ajuste de las ecuaciones probadas fue estadísticamente adecuado (Tabla 4 y Tabla 5), al generar, en la mayoría de los casos, coeficientes de determinación $\left(R^{2}\right.$ y $\left.R^{2} a d j\right)$ altos, suma de cuadrados del error (SCE), raíz del error cuadrático medio (RECM), sesgo promedio absoluto $(\bar{E})$ y errores estándar de los parámetros pequeños. Para todos los ajustes, los estimadores de los parámetros fueron significativos $(P<0.05)$.

TABLA 3. Distribuciones rechazadas por su falta de ajuste a la fdp Weibull de acuerdo con diferentes niveles de significancia.

\begin{tabular}{ccc}
\hline$\alpha$ & $\begin{array}{c}\text { Número de distribuciones } \\
\text { rechazadas }\end{array}$ & $\begin{array}{c}\text { Proporción } \\
(\%)\end{array}$ \\
\hline 0.05 & 8 & 6.06 \\
0.10 & 13 & 9.85 \\
0.20 & 21 & 15.91 \\
\hline
\end{tabular}

"Proporción calculada a partir de 132 distribuciones diamétricas.

TABLA 4. Estadísticas de bondad de ajuste de las ecuaciones que predicen los parámetros de la fdp Weibull en función de los atributos del rodal.

\begin{tabular}{ccccccc}
\hline Ecuación & $S C E$ & $R E C M$ & $R^{2}$ & $R^{2}{ }_{a d j}$ & $\bar{E}$ & AlC \\
\hline a.E1 & 112.279 & 1.010 & 0.859 & 0.859 & 0.041 & 3.271 \\
a.E2 & 36.248 & 0.582 & 0.955 & 0.953 & $3.7 \mathrm{E}-15$ & -116.226 \\
b.E1 & 217.900 & 1.427 & 0.916 & 0.914 & $3.5 \mathrm{E}-15$ & 82.870 \\
b.E2 & 184.300 & 1.313 & 0.929 & 0.927 & $2.8 \mathrm{E}-15$ & 64.281 \\
c.E1 & 41.568 & 0.620 & 0.235 & 0.220 & 0.023 & -93.228 \\
c.E2 & 36.358 & 0.583 & 0.330 & 0.312 & 0.024 & -115.889 \\
\hline
\end{tabular}

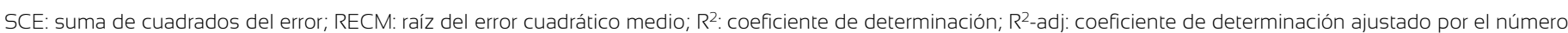
de parámetros; $\overline{\mathrm{E}}$ : sesgo promedio absoluto; AIC: criterio de información de Akaike. 
TABLA 5. Parámetros estimados de las ecuaciones que predicen los parámetros de la fdp Weibull en función de los atributos del rodal.

\begin{tabular}{|c|c|c|c|c|c|c|c|c|c|}
\hline$E c$ & & Estimación & $\begin{array}{c}\text { Error } \\
\text { estándar }\end{array}$ & $P>|t|$ & $E c$ & & Estimación & $\begin{array}{c}\text { Error } \\
\text { estándar }\end{array}$ & $P>|t|$ \\
\hline \multirow{4}{*}{ a.E1 } & \multirow{4}{*}{ ßo } & \multirow{4}{*}{0.8817} & \multirow{4}{*}{0.015} & \multirow{4}{*}{$<0.0001$} & \multirow{4}{*}{ a.E2 } & $\beta_{0}$ & 1.0755 & 0.137 & $<0.0001$ \\
\hline & & & & & & $\beta_{l}$ & -0.7646 & 0.022 & $<0.0001$ \\
\hline & & & & & & $\beta_{2}$ & 1.7209 & 0.065 & $<0.0001$ \\
\hline & & & & & & $\beta_{3}$ & -0.8566 & 0.058 & $<0.0001$ \\
\hline \multirow{4}{*}{ b.E1 } & $\beta_{0}$ & -4.1956 & 0.514 & $<0.0001$ & \multirow{4}{*}{ b.E2 } & $\beta_{0}$ & -6.0631 & 0.486 & $<0.0001$ \\
\hline & $\beta_{l}$ & 2.9085 & 0.205 & $<0.0001$ & & $\beta_{1}$ & 2.6496 & 0.183 & $<0.0001$ \\
\hline & $\beta_{2}$ & -1.4985 & 0.136 & $<0.0001$ & & $\beta_{2}$ & -0.0360 & 0.003 & $<0.0001$ \\
\hline & $\beta_{3}$ & 0.8536 & 0.028 & $<0.0001$ & & $\beta_{3}$ & 0.8074 & 0.023 & $<0.0001$ \\
\hline \multirow{4}{*}{ c.E1 } & $\beta_{0}$ & -0.3400 & 0.062 & $<0.0001$ & \multirow{4}{*}{ c.E2 } & $\beta_{0}$ & -0.2908 & 0.060 & $<0.0001$ \\
\hline & $\beta_{1}$ & 0.3886 & 0.069 & $<0.0001$ & & $\beta_{1}$ & 0.3272 & 0.067 & $<0.0001$ \\
\hline & \multirow[b]{2}{*}{$\beta_{2}$} & \multirow[b]{2}{*}{0.0454} & \multirow[b]{2}{*}{0.004} & \multirow[b]{2}{*}{$<0.0001$} & & $\beta_{2}$ & 0.0559 & 0.004 & $<0.0001$ \\
\hline & & & & & & $\beta_{3}$ & -0.3277 & 0.084 & 0.0002 \\
\hline
\end{tabular}

Desde el punto de vista estadístico, las ecuaciones a.E2, b.E2 y c.E2 predicen satisfactoriamente los parámetros de localización, escala y forma de la fdp Weibull. Las ecuaciones ajustadas en conjunto con las funciones de altura y volumen hicieron posible predecir el rendimiento maderable en términos implícitos, que consiste en determinar las frecuencias de árboles por categoría diamétrica (Fig. 2 y Fig. 3), es importante resaltar que, para este método, el diámetro medio cuadrático se derivó de la proyección del área basal y número de árboles por hectárea del sistema explícito para P. patula (Santiago-García et al., 2017).

\section{Predicción de percentiles}

Las ecuaciones ajustadas para proyectar el diámetro mínimo, diámetro medio cuadrático y las ecuaciones de predicción de los percentiles 55 y 85 de la distribución diamétrica, generaron indicadores de bondad de ajuste satisfactorios (Tabla 6 y Tabla 7), es decir, coeficientes de determinación $\left(\mathrm{R}^{2} \mathrm{y} \mathrm{R}^{2}\right.$ adj) altos, suma de cuadrados del error (SCE), raíz del error cuadrático medio (RECM), sesgo promedio absoluto $(\bar{E})$ y errores estándar de los parámetros pequeños, además, todos los parámetros fueron diferentes de cero $(P<0.05)$.

La predicción de percentiles y el método de momentos, así como las ecuaciones de proyección del crecimiento en diámetro mínimo y diámetro medio cuadrático del rodal, permitieron recuperar los parámetros de la fdp Weibull en el tiempo, esto fue posible debido a que dichos parámetros dependen de las variables de estado, por lo que, al proyectarlas en el tiempo, se recupera o conoce el valor de los parámetros a una edad de interés (Prodan, Peters, Cox y Real, 1997; Poudel y Cao, 2013; Santiago-García et al., 2014), es importante mencionar que por la estrecha relación entre momentos (o percentiles en su caso) y los atributos del rodal, esta técnica ha brindado modelos con ajustes satisfactorios (Borders y Patterson, 1990). 


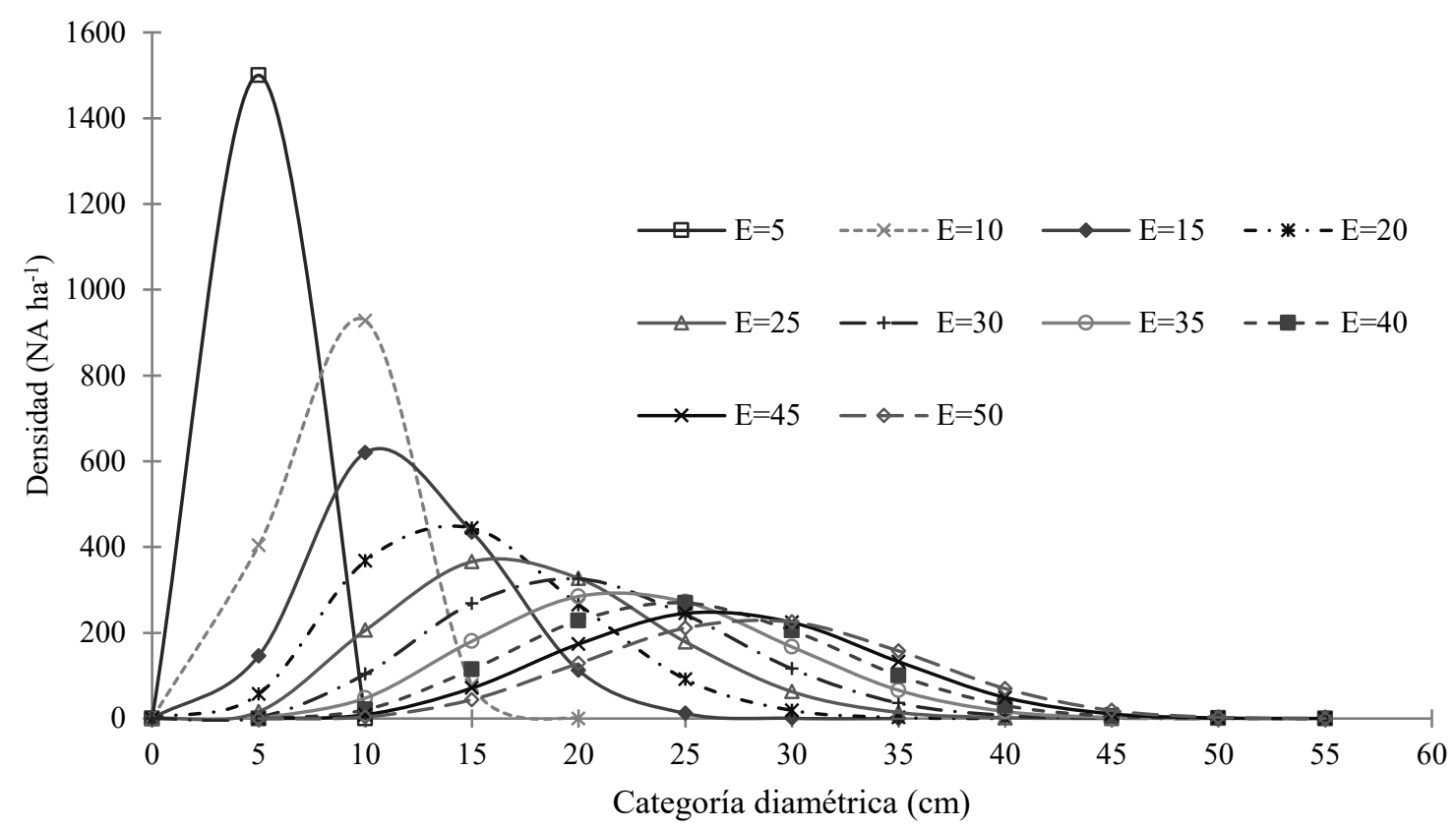

FIgURA 2. Número de árboles $\left(\mathrm{Na} \mathrm{ha}^{-1}\right)$ por categoría diamétrica para cada periodo de proyección (E: edad) obtenido con predicción de parámetros de la fdp Weibull, considerando la calidad de sitio promedio (IS = $29 \mathrm{~m}$ ) y la densidad inicial de 1500 árboles por hectárea a la edad de cinco años.

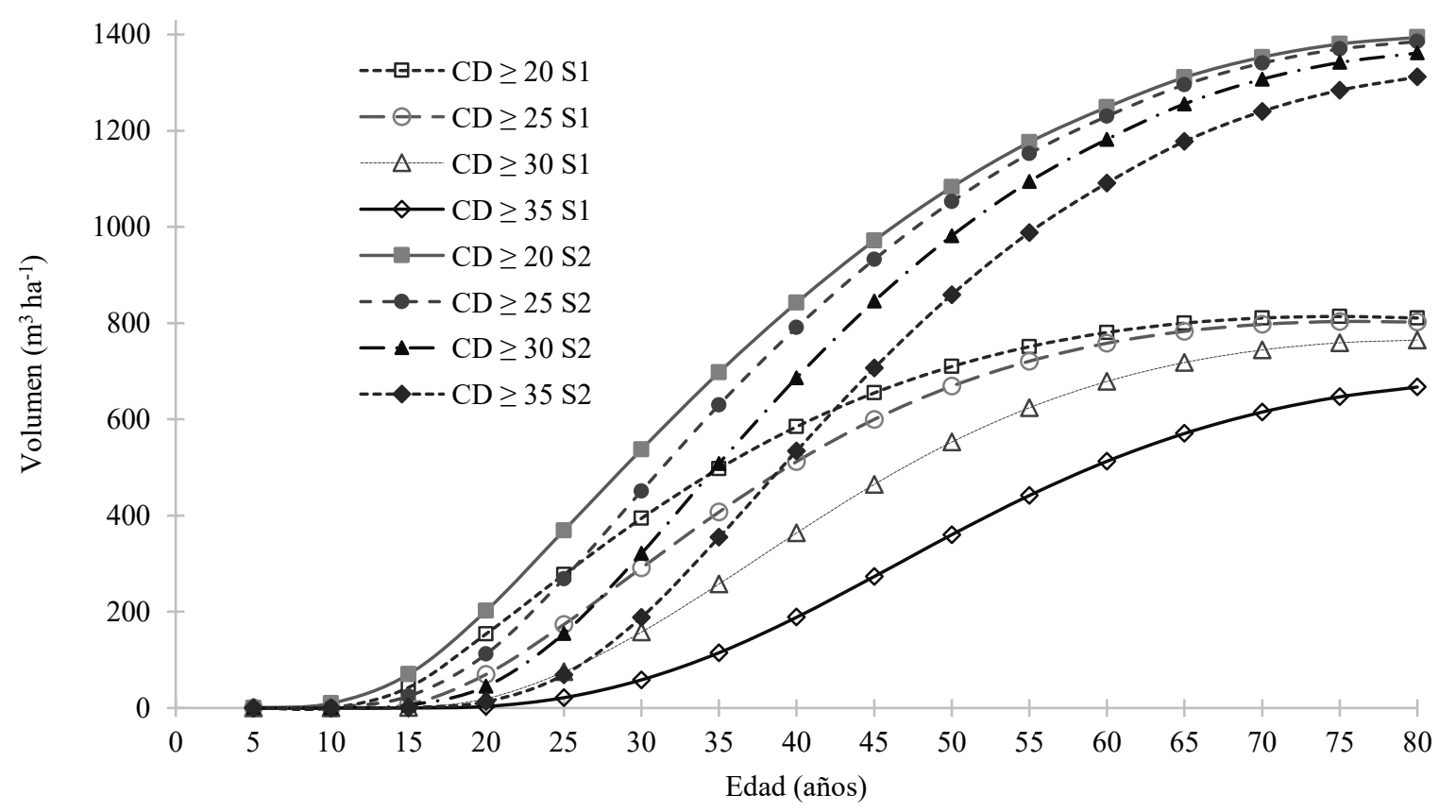

FIGURA 3. Curvas de rendimiento volumétrico estimadas para diferentes categorías diamétricas (CD) a través del tiempo, considerando dos métodos implícitos (S1: predicción de parámetros, S2: predicción de percentiles). 
TABLA 6. Estadísticas de bondad de ajuste de las ecuaciones que predicen los percentiles 55 y 85 de la distribución diamétrica del rodal, el diámetro mínimo y el diámetro medio cuadrático.

\begin{tabular}{ccccccc}
\hline Ecuación & SCE & RECM & $R^{2}$ & $R^{2}$ adj & $\bar{E}$ & AlC \\
\hline$p 55$ & 690.100 & 2.304 & 0.908 & 0.908 & 0.090 & 222.333 \\
$p 85$ & 552.800 & 2.062 & 0.959 & 0.959 & 0.034 & 193.050 \\
$D$ min 2 & 7.141 & 0.334 & 0.986 & 0.986 & 0.003 & -142.767 \\
$D q_{1}$ & 258.400 & 2.009 & 0.925 & 0.924 & 0.043 & 96.080 \\
$D q_{2}$ & 29.382 & 0.672 & 0.991 & 0.991 & 0.059 & -47.412 \\
\hline
\end{tabular}

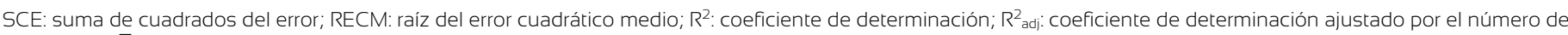
parámetros; $\overline{\mathrm{E}}$ : sesgo promedio absoluto; AIC: criterio de información de Akaike.

TABLA 7. Parámetros estimados de las ecuaciones que predicen los percentiles 55 y 85 de la distribución diamétrica del rodal, el diámetro mínimo y el diámetro medio cuadrático.

\begin{tabular}{ccccc}
\hline Ecuación & Parámetro & Estimación & Error estándar & $P>|t|$ \\
\hline \multirow{2}{*}{$p 55$} & $\alpha$ & 0.7532 & 0.065 & $<0.0001$ \\
& $\beta$ & 1.0813 & 0.028 & $<0.0001$ \\
\multirow{2}{*}{$p 85$} & $\alpha$ & 1.1051 & 0.063 & $<0.0001$ \\
& $\beta$ & 1.0563 & 0.018 & $<0.0001$ \\
Dmin 2 & $\beta_{1}$ & 0.1911 & 0.050 & 0.0003 \\
& $\beta_{2}$ & 1.9149 & 0.453 & $<0.0001$ \\
$D q_{1}$ y $D q_{2}$ & $\beta_{0}$ & 2.2347 & 0.073 & $<0.0001$ \\
& $\beta_{1}$ & -0.00201 & 0.000 & $<0.0001$ \\
& $\beta_{2}$ & 0.0419 & 0.003 & $<0.0001$ \\
\hline
\end{tabular}

Con las ecuaciones ajustadas fue posible determinar la proporción de árboles para cada categoría diamétrica en el rodal (Fig. 4). Para estimar el rendimiento maderable con el método de recuperación de parámetros mediante predicción de percentiles se requieren los mismos modelos adicionales (ecuaciones de altura-diámetro y volumen individual) usados en el sistema de predicción de parámetros, al interrelacionar estas ecuaciones fue posible la predicción del rendimiento maderable por categoría diamétrica (Fig. 3 y Fig. 4).

\section{Comparación de los métodos}

Al comparar gráficamente el rendimiento maderable derivado de un modelo de totalidad del rodal (predicción explícita) diseñado para Pinus patula (Santiago-García et al., 2017) y los rendimientos predichos implícitamente (Fig. 5), es evidente la diferencia entre las estimaciones, porque el rendimiento maderable total predicho por el método de predicción de percentiles es mayor al volumen explícito, mientras que el método de predicción de parámetros predice el rendimiento maderable de forma similar al sistema explícito.

En una predicción implícita del rendimiento existen más errores acumulados que en una predicción explícita (porque se usan más ecuaciones para realizar la predicción implícita), esta última se usa para corregir las estimaciones de la primera. El procedimiento de corrección consiste en distribuir las diferencias de ambas proyecciones en área basal o volumen de acuerdo con la participación de cada categoría diamétrica en estas variables. El factor de corrección $(F C)$ se calculó como:

$$
F C=\frac{V E}{V I}
$$




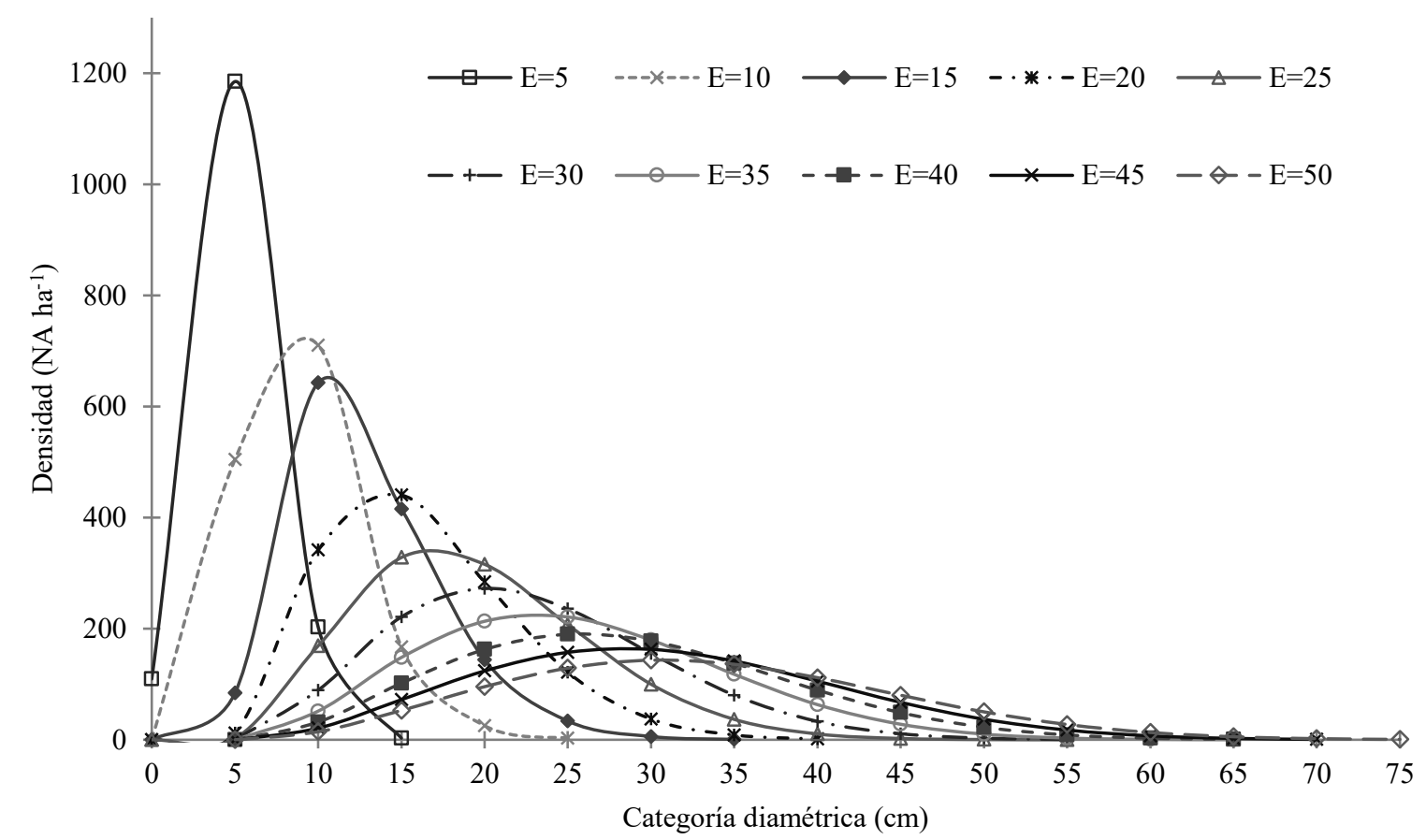

FIGURA 4. Número de árboles $\left(\mathrm{N} A \mathrm{ha}^{-1}\right)$ por categoría diamétrica para cada periodo de proyección (E: edad) obtenido con predicción de percentiles, considerando la calidad de sitio promedio (IS $=29 \mathrm{~m}$ ) y densidad inicial de 1500 árboles por hectárea a la edad de cinco años.

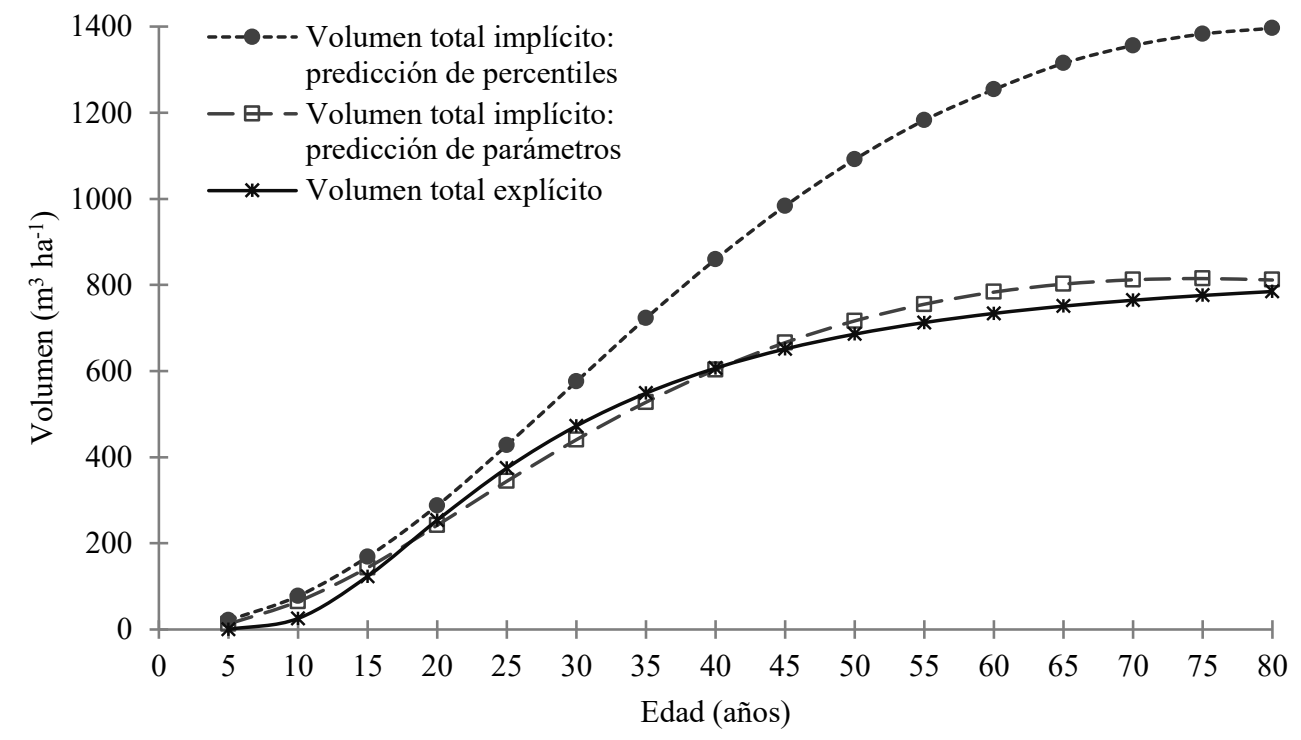

FIGURA 5. Comparación del volumen total explícito proyectado a un $I S=29$ y $N A_{1}=1500$ árboles por hectárea con el volumen total implícito predicho por el método de predicción de parámetros y predicción de percentiles. 
donde:

$V E$ : volumen total explícito $\left(\mathrm{m}^{3} \mathrm{ha}^{-1}\right)$

$V I$ : volumen total implícito $\left(\mathrm{m}^{3} \mathrm{ha}^{-1}\right)$ (Magaña, Torres, Rodríguez, Aguirre y Fierros, 2008).

Una vez obtenido este valor, se multiplica por el volumen de cada categoría diamétrica.

El FC (Tabla 8) se estimó para ambas predicciones implícitas y se obtuvieron las curvas de rendimiento volumétrico corregidas por dicho factor (Fig. 6); de esta manera, la estimación es más realista y ambos sistemas tienden a ser similares.

Con el factor de corrección, las estimaciones son equivalentes, aunque con el sistema basado en predicción de percentiles, el volumen estimado por categoría diamétrica es ligeramente mayor que el estimado por el sistema basado en predicción de parámetros.

TABLA 8. Factores de corrección para el volumen implícito generado mediante volumen explícito.

\begin{tabular}{cccccccccccc}
\hline \multirow{2}{*}{ Edad } & \multirow{2}{*}{ VTE } & \multicolumn{2}{c}{ VTI } & \multicolumn{2}{c}{$F C$} & \multirow{2}{*}{ Sdad } & \multirow{2}{*}{ VTE } & \multicolumn{2}{c}{ STI } & \multicolumn{3}{c}{$F C$} & S1 & S2 \\
\hline 5 & 0.23 & 12.79 & 21.41 & 0.02 & 0.01 & 45 & 651.38 & 666.12 & 983.48 & 0.98 & 0.66 \\
10 & 25.64 & 65.18 & 77.62 & 0.39 & 0.33 & 50 & 685.82 & 716.61 & 1091.49 & 0.96 & 0.63 \\
15 & 123.51 & 143.73 & 168.84 & 0.86 & 0.73 & 55 & 712.71 & 755.29 & 1182.55 & 0.94 & 0.60 \\
20 & 253.98 & 242.23 & 287.85 & 1.05 & 0.88 & 60 & 733.94 & 783.39 & 1253.83 & 0.94 & 0.59 \\
25 & 374.71 & 344.17 & 428.00 & 1.09 & 0.88 & 65 & 750.87 & 801.91 & 1314.62 & 0.94 & 0.57 \\
30 & 472.72 & 440.66 & 575.75 & 1.07 & 0.82 & 70 & 764.53 & 811.99 & 1355.94 & 0.94 & 0.56 \\
35 & 548.67 & 527.68 & 722.81 & 1.04 & 0.76 & 75 & 775.65 & 814.84 & 1382.69 & 0.95 & 0.56 \\
40 & 606.81 & 603.16 & 859.50 & 1.01 & 0.71 & 80 & 784.78 & 811.46 & 1396.14 & 0.97 & 0.56 \\
\hline
\end{tabular}

VTE: volumen total explícito; VTI: volumen total implícito; FC: factor de corrección: Sl: predicción de parámetros, S2: predicción de percentiles.

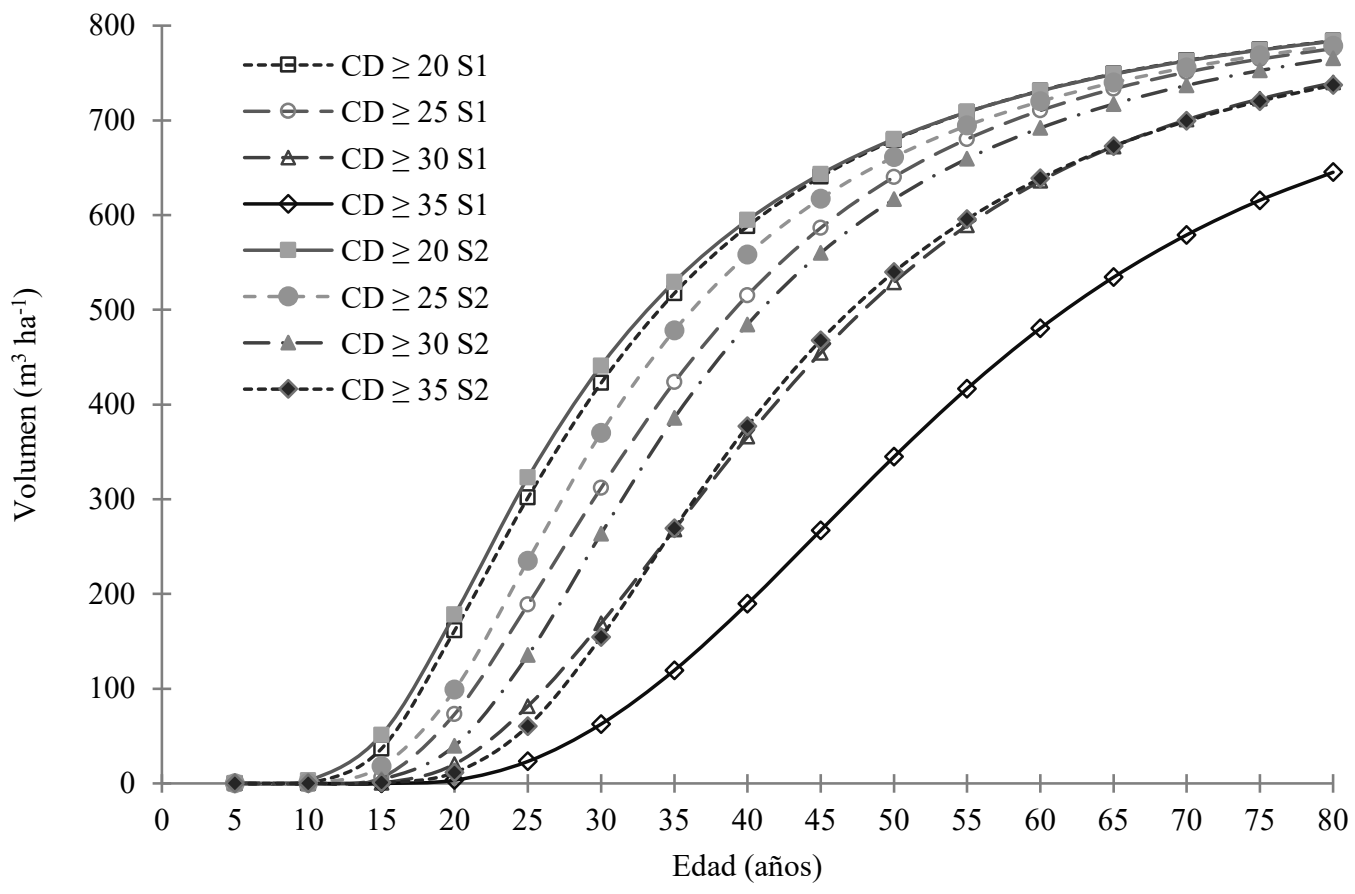

FIGURA 6. Curvas de rendimiento volumétrico corregidas para diferentes categorías diamétricas (CD) a través del tiempo, considerando dos métodos implícitos (S1: predicción de parámetros, S2: predicción de percentiles). 


\section{DISCUSIÓN}

\section{Predicción de parámetros de la fdp Weibull}

La ecuación para predecir el parámetro de localización (a) seleccionada por su bondad de ajuste fue:

$$
\begin{gathered}
a=1.0755-0.7646 \cdot b+1.7209 \cdot D m \\
-0.8566 \cdot D q
\end{gathered}
$$

Esta ecuación indica la relación existente entre el parámetro de localización (a), diámetro promedio $(D m)$ y el diámetro medio cuadrático $(D q)$, este resultado coincide con el registrado por De la Fuente et al. (1998), para rodales coetáneos de Pinus rudis Endl. en Pueblos Mancomunados (Oaxaca), y por Torres-Rojo, Magaña-Torres y AcostaMireles (2000), para P. montezumae Lamb. en San Juan Tetla (Puebla), ya que estos autores utilizaron tanto el $D m$ como el $D q$ para estimar el parámetro $a$, además, estas variables son fundamentales para caracterizar la distribución diamétrica del rodal.

Con respecto al parámetro de escala (b), la ecuación de predicción seleccionada fue:

$$
\begin{array}{r}
b=-6.0631+2.6496 \cdot c-0.0360 \\
\cdot D \min ^{2}+0.8074 \cdot D q
\end{array}
$$

esta es parcialmente similar a la propuesta por Quiñonez, De los Santos, Cruz, Velázquez y Ramírez (2015), quienes para estimar las distribuciones diamétricas en masas mezcladas de especies del género Pinus, sugieren como variables predictoras de este parámetro el diámetro mínimo, el diámetro medio cuadrático y el diámetro promedio del rodal.

El parámetro de forma $(c)$ fue predicho de mejor manera con la ecuación:

$$
\begin{aligned}
c=- & 0.2908 \cdot D q+0.3272 \cdot D m+ \\
& 0.0559 \cdot I S-0.3277 \cdot C a
\end{aligned}
$$

Haan (1986) indicó que la técnica de momentos relaciona el coeficiente de asimetría $(C a)$ con el parámetro $c$, de acuerdo con esta condición Návar-Cháidez (2009) propuso predecir este parámetro en función del Ca; Magaña, Torres, Rodríguez, Aguirre y Fierros (2008) obtuvieron que para $P$. rudis Endl. en Aloapan (Oaxaca), la predicción del parámetro $c$ es satisfactoria al considerar como variables independientes el diámetro medio cuadrático, la calidad de sitio (IS), el número de árboles y la altura promedio. En tanto que Quiñonez, De los Santos, Cruz, Velázquez y Ramírez (2015) sugieren como variables independientes el diámetro promedio, el diámetro medio cuadrático y el diámetro mínimo.

\section{Predicción de percentiles}

En la predicción de la distribución diamétrica, la elección de los percentiles es crucial y depende de la muestra, por lo que una pareja de percentiles al estar más centrada ayuda a reducir el sesgo en la predicción (Santiago-García et al., 2014). En este estudio, los percentiles 55 y 85 permitieron calcular el parámetro $c$ de la fdp Weibull y obtener estimaciones adecuadas de la distribución diamétrica de los rodales de Pinus patula. Pienaar y Rheney (1993) y Montero y Fierros (2000) usaron los percentiles 24 y 93 para calcular el parámetro c. García, Cañadas y Montero (2002) señalan que con la pareja de percentiles 40 y 82 pueden conseguirse ajustes eficaces. En tanto que, Santiago-García et al. (2014) obtuvieron resultados satisfactorios con los percentiles 50 y 90 de la distribución diamétrica.

\section{Comparación de los métodos}

En la estimación del rendimiento maderable, el sistema de predicción de percentiles generó valores más altos que el sistema de predicción de parámetros, esto, debido a que las distribuciones diamétricas del rodal obtenidas con predicción de percentiles abarcaron más valores con probabilidad de ocurrencia (Fig. 4). En el caso del método de predicción de parámetros, el intervalo de las clases diamétricas estimadas para $P$. patula abarcó de $5 \mathrm{~cm}$ a $60 \mathrm{~cm}$ en un intervalo de edad de 5 años a 80 años, en tanto que, con el método de predicción de percentiles se obtuvieron valores de diámetro menores a $2.5 \mathrm{~cm}$ e iguales a $90 \mathrm{~cm}$ en el mismo intervalo de edad. Embrechts, Klüppelberg y 
Mikosch (1997) argumentan que las distribuciones subexponenciales como la Weibull son de colas pesadas, porque tienden a 0 lentamente. Por tanto, tienen más probabilidad de encontrar valores extremos repartidas en ellas. En este caso, la probabilidad mayor ocurrió para las categorías diamétricas superiores en el sistema de predicción de percentiles, en consecuencia, se obtuvieron valores más altos de rendimiento maderable.

Las estimaciones implícitas de rendimiento maderable se compararon contra un modelo de volumen explícito de $P$. patula existente en el área de estudio (Santiago-García et al., 2017). Se considera que este tipo de modelos tienen menor error en la estimación del rendimiento de rodales coetáneos, porque el nivel de resolución es directamente en $\mathrm{m}^{3} \mathrm{ha}^{-1}$ y se reconoce que representan un buen compromiso entre precisión y generalidad (García, 1988; Vanclay, 1994; Diéguez-Aranda, Castedo, Álvarez y Rojo, 2006). Por esta razón, las estimaciones realizadas con el método de predicción de parámetros de la fdp Weibull en función de las variables del rodal son más realistas que el sistema basado en predicción de percentiles, ya que las estimaciones fueron similares a las obtenidas con el modelo explícito.

En el presente estudio se estimó, con el sistema implícito de predicción de parámetros, que el volumen del fuste total con corteza (Tabla 8) a los 15 años fue de 143.73 $\mathrm{m}^{3} \mathrm{ha}^{-1}$; en tanto que, con el sistema de predicción de percentiles, se estimó $168.84 \mathrm{~m}^{3} \mathrm{ha}^{-1}$; para el área basal se estimó $16.99 \mathrm{~m}^{2} \mathrm{ha}^{-1}$ y $19.21 \mathrm{~m}^{2} \mathrm{ha}^{-1}$ para los sistemas de predicción de parámetros y predicción de percentiles, respectivamente, al considerar una densidad de 1326 árboles por hectárea. Al respecto, Arteaga (2003) obtuvo que el rendimiento en volumen de una plantación de $P$. patula de 14.6 años en Perote, Veracruz fue de $32.39 \mathrm{~m}^{3}$ ha1 de fuste total sin corteza con una densidad de 976 árboles $\mathrm{ha}^{-1}$, área basal de $9.21 \mathrm{~m}^{2} \mathrm{ha}^{-1} \mathrm{y}$ altura media de $6.53 \mathrm{~m}$. Por su parte, Santiago-García et al. (2014) estimaron, para los 15 años, cerca de $150 \mathrm{~m}^{3} \mathrm{ha}^{-1}$ de fuste total con corteza, de acuerdo con una densidad inicial de 1400 árboles por hectárea a los cinco años de edad e índice de sitio de $29 \mathrm{~m}$ a la edad base de 40 años para rodales coetáneos de $P$. patula en el ejido La Mojonera, Municipio de Zacualtipán, Hidalgo.

Los sistemas de crecimiento implícitos presentados en este artículo son instrumentos que apoyan la planificación forestal, al permitir definir la distribución de productos maderables, los regímenes silvícolas que generen estructuras residuales deseables, o los tipos, intensidad y frecuencia de aclareos. Estos sistemas pueden complementarse con una ecuación de ahusamientovolumen, que permita realizar la distribución de productos a nivel árbol individual.

\section{CONCLUSIONES}

Los sistemas estudiados permiten obtener predicciones del rendimiento maderable por categoría diamétrica a nivel de unidad de superficie, son sencillos de implementar y permiten caracterizar los productos que se tendrán en el bosque en un determinado momento. Con los modelos de proyección de las variables dasométricas del rodal se recuperaron los parámetros de la función de distribución de probabilidades Weibull a diferentes edades de interés. Para este estudio, el sistema basado en predicción de parámetros arrojó resultados satisfactorios. Estos modelos permiten representar la estructura diamétrica de un rodal en términos numéricos a través de una tabla de rendimiento, la cual muestra el cambio de estructura de un rodal a medida que cambia la edad, por lo que constituyen una herramienta valiosa para planificar el manejo de los rodales de Pinus patula en Ixtlán de Juárez, Oaxaca, México. La precisión e intervalo de validez de los sistemas propuestos puede mejorarse al aumentar el número de mediciones y reajustar el sistema con cada remedición.

\section{RECONOCIMIENTOS}

Este trabajo se llevó a cabo con el apoyo de la Comunidad de Ixtlán de Juárez y del Programa para el Desarrollo Profesional Docente, para el Tipo Superior, [Prodep]. Proyecto: IDCA 24332 UNSIJ-CA-6 "Estructura, Dinámica, Producción y Ecología de Especies Forestales en la Sierra Norte de Oaxaca". 


\section{REFERENCIAS}

Alder, D. (1980). Estimación del volumen forestal y predicción del rendimiento con referencia especial a los trópicos (Vol. 2). Roma, Italia: Organización de las Naciones Unidas.

Amaro, A., Reed, D., Tomé, M., \& Temido, I. (1998). Modelling dominant height growth: Eucalyptus plantations in Portugal. Forest Science, 44(1), 37-46. doi: 10.1093/forestscience/44.1.37

Arteaga, B. (2003). Evaluación dasométrica de una plantación de Pinus spp. en Perote, Veracruz, México. Foresta Veracruzana, 5(1), 27-32.

Assmann, E. (1970). The principles of forest yield study. Pergamon Press: Oxford.

Bailey, R. \& Dell, T. (1973). Quantifying diameter distributions with the Weibull function. Forest Science, 19(2), 97-104. doi: 10.1093/forestscience/19.2.97

Borders, B. \& Patterson, W. (1990). Projecting stands tables: A comparison of the Weibull diameter distribution method, a Percentile-Based projection method, and a basal area growth projection method. Forest Science, 36(2), 413-424. doi: 10.1093/forestscience/36.2.413

Borders, B., Souter, R., Bailey, R., \& Ware, K. (1987). Percentile-based distributions characterize forest stand tables. Forest Science, 33, 570-576. doi: 10.1093/forestscience/33.2.570

Cao, C. (2004). Predicting parameters of Weibull function for modeling diameter distribution. Forest Science, 50(5), 682-685. doi: $10.1093 /$ forestscience/ 50.5 .682

Clutter, J. L., Forston, J. C., Pienaar, L. V., Brister, G. H., \& Bailey, R. L. (1983). Timber Management: A Quantitative Approach. Nueva York, Estados Unidos: John Wiley \& Sons, Inc.

De la Fuente E., A., Velásquez M., A., Torres R., J. M., Ramírez M., H., Rodríguez F., C., \& Trinidad S., A. (1998). Predicción del crecimiento y rendimiento de Pinus rudis Endl., en Pueblos Mancomunados, Ixtlán, Oaxaca. Revista Ciencia Forestal en México, 23(84), 3-8.

Diéguez-Aranda, U., Castedo D., F., Álvarez G., J. G., \& Rojo A., A. (2006). Dynamic growth model for Scots pine (Pinus sylvestris L.) plantations in Galicia (north-western Spain). Ecological Modelling, 191:225-242. doi: 10.1016/j.ecolmodel.2005.04.026

Embrechts, P., Klüppelberg, C., \& Mikosch, T. (1997). Modelling Extremal Events for Insurance and Finance. New York: Springer.

Galán L., R., De los Santos P., H. M., \& Valdez H., J. I. (2008). Crecimiento y rendimiento maderable de Cedrela odorata L. y Tabebuia donnell-smithii Rose en San José Chacalapa, Pochutla, Oaxaca. Madera y Bosques, 14(2), 65-82. doi: $10.21829 /$ myb.2008.1421213
García, G., C., Cañadas, N., \& Montero, G. (2002). Modelización de la distribución diamétrica de las masas de Pinus pinea L. de Valladolid (España) mediante la función Weibull. Investigación Agraria. Sistemas y Recursos Forestales, 11(2), 263-282.

García, O. (1988). Growth modeling-a (re)view. New Zealand Journal of Forestry, 33(3),14-17.

Gómez-García, E., Crecente-Campo, F., Pérez-Rodríguez, F., \& Diéguez-Aranda, U. (2013). Ajuste de la función Weibull biparamétrica a distribuciones diamétricas de rodales regulares de Quercus robur L. en Galicia. En V. Gasteiz (Comp.), Montes: Servicios y desarrollo rural. Sociedad Española de Ciencias Forestales.

Haan, C. T. (1986). Statistical Methods in Hydrology. Iowa: State Press.

Hafley, W. L. \& Schreuder H., T. (1977). Statistical distributions for fitting diameter and height data in even-aged stands. Canadian Journal of Forest Research, 7(3), 481-487. doi: 10.1139/x77-062

Hirigoyen, A. \& Rachid, C. (2014). Selección de funciones de distribución de frecuencias diamétricas, para Pinus taeda, Eucalyptus globulus y Eucalyptus dunnii en Uruguay. Bosque, 35(3), 369-376. doi: 10.4067/S0717-92002014000300011

Hyink, D. M. \& Moser, J. W. (1983). A generalized framework for projecting forest yield and stand structure using diameter distributions. Forest Science, 29(1), 85-95. doi: 10.1093/forestscience/29.1.85

López-Villegas, M. F., Santiago-García, W., Quiñonez-Barraza, G., Suárez-Mota, M. E., Santiago-Juárez, W., \& Santiago-García, E. (2017). Ecuaciones globales y locales de altura-diámetro de 12 especies de interés comercial en bosques manejados. Revista Mexicana de Agroecosistemas, 4(2), 113-126.

Magaña T., O. S., Torres R., J. M., Rodríguez F., C., Aguirre D., H., \& Fierros G., A. M. (2008). Predicción de la producción y rendimiento de Pinus rudis Endl., en Aloapan, Oaxaca. Madera y Bosques, 14(1), 5-19. doi: 10.21829/myb.2008.1411214

Maldonado A., D. \& Návar Ch., J. (2002). Ajuste y predicción de la distribución Weibull a las estructuras diamétricas de plantaciones de pino de Durango, México. Madera y Bosques, 8(1), 61-72. doi: $10.21829 /$ myb.2002.811306

Maltamo, M., Puumalainen, J., \& Päivinen, R. (1995). Comparison of Beta and Weibull functions for modeling basal area diameter distribution in stands of Pinus sylvestris and Picea abies. Scandinavian Journal of Forest Research, 1-4, 284-295. doi: 10.1080/02827589509382895

Montero M., M. \& Fierros G., A. M. (2000). Predicción del crecimiento de Pinus caribaea var. hondurensis Barr y Golf. en "La Sabana", 
Oaxaca, México. Comunicación Técnica. Revista Forestal Centroamericana, 32, 20-25.

Návar-Cháidez, J. (2009). Estimaciones empíricas de parámetros de la distribución Weibull en bosques nativos del norte de México. Revista Forestal Latinoamericana, 24(2), 51-68.

Pece, M. G., G. de Benítez, C., \& J. de Galíndez, M. (2000). Uso de la función Weibull para modelar distribuciones diamétricas en una plantación de Melia azedarach. Revista Forestal Venezolana, 44(2), 49-52.

Pérez-López, E., Santiago-García, W., Quiñonez-Barraza, G., Rodríguez-Ortiz, G., \& Ruiz-Aquino, F. (2017). Ecuaciones dinámicas: Altura dominante e índice de sitio para Pinus patula Schiede ex Schlechtendal \& Chamisso. En V. J. C. Vinay, V. A. Esqueda E., O. H. Tosquy V., A. Ríos U., M. V. Vázquez H., \& C. Perdomo M. (Comps.). Avances en Investigación Agricola, Pecuaria, Forestal, Acuicola, Pesquería, Desarrollo rural, Transferencia de tecnología, Biotecnología, Ambiente, Recursos naturales y Cambio Climático-2017 (pp. 1125-1133). Medellín, Ver., México: Inifap. CP. UV. UACH. AVC. Itboca. ITUG. ITSH. UPH.

Pienaar, L. V. \& Rheney, J. W. (1993). Yield prediction for mechanically site-prepared slash pine plantations in the southeastern coastal plain. Southern Journal of Applied Forestry, 17(4), 163-173. doi: 10.1093/sjaf/17.4.163

Poudel, K. P. \& Cao, Q. V. (2013). Evaluation of methods to predict Weibull parameters for characterizing diameter distributions. Forest Science, 59(2), 243-252. doi: 10.5849/forsci.12-001

Prodan, M., Peters, R., Cox, F., \& Real, P. (1997). Mensura forestal. San José, Costa Rica: IICA y GTZ.

Quevedo, A., Moret, A. Y., \& Jerez, M. (2003). Comparación de métodos de ajuste de funciones de probabilidad para distribuciones diamétricas en plantaciones de teca. Revista Forestal Venezolana, 47(2), 53-60.

Quiñonez B., G., De los Santos P., H. M., Cruz C., F., Velázquez M., A., \& Ramírez V., G. (2015). Modelación dinámica de distribuciones diamétricas en masas mezcladas de Pinus en Durango, México. Madera y Bosques, 21(2), 59-71. doi: 10.21829/myb.2015.212445

Rennolls, K., Geary, D. N., \& Rollinson, T. J. D. (1985). Characterizing diameter distributions by the use of the Weibull distribution. Forestry, 58(1), 57-66. doi: 10.1093/forestry/58.1.57
Reynolds, M. R., Burk, T. E., \& Huang, W. C. (1988). Goodness-of-fit test and model selection procedures for diameter distribution models. Forest Science, 34(2), 373-399. doi: 10.1093/forestscience/34.2.373

Rodríguez-Justino, R. (2017). Sistemas compatibles de cubicación de árboles individuales para dos especies de interés comercial en Ixtlán de Juárez, Oaxaca. Tesis de maestría. Universidad de la Sierra Juárez. Ixtlán de Juárez, Oaxaca, México.

Santiago-García, W., De los Santos-Posadas, H. M., Ángeles-Pérez, G., Corral Rivas, J. J., Valdez-Lazalde, J. R., \& Del Valle-Paniagua, D. H. (2014). Predicción del rendimiento maderable de Pinus patula Schl. et Cham. a través de modelos de distribución diamétrica. Agrociencia, 48(1), 87-101.

Santiago-García, W., Pérez-López, E., Quiñonez-Barraza, G., Rodríguez-Ortiz, G., Santiago-García, E., Ruiz-Aquino, F., \& Tamarit-Urias, J.C. (2017). A dynamic system of growth and yield equations for Pinus patula. Forests, 8(12), 465. doi: $10.3390 /$ f 8120465

SAS Institute Inc. (2011). SAS/ETS® 9.3 User's Guide. Cary, NC:, SAS Institute Inc.

Servicios técnicos forestales de Ixtlán de Juárez [STF] (2015). Programa de manejo forestal para el aprovechamiento y conservación de los recursos forestales maderables de Ixtlán de Juárez: Ciclo de corta 2015-2024. Ixtlán de Juárez, Oaxaca, México.

Sokal, R. \& Rohlf, J. (1979). Biometría: principios y métodos estadísticos en la investigación biológica. Madrid: $\mathrm{H}$. Blume.

Torres-Rojo, J. M., Acosta M., M., \& Magaña T., O. S. (1992). Métodos para estimar los parámetros de la función Weibull y su potencial para ser predichos a través de atributos del rodal. Agrociencia. Serie Recursos Naturales Renovables, 2, 57-76.

Torres-Rojo, J. M., Magaña-Torres, O. S., \& Acosta-Mireles, M. (2000). Metodología para mejorar la predicción de parámetros de la distribuciones diamétricas (Ensayo). Agrociencia, 34(5), 627-637.

Vanclay, J. K. (1994). Modelling forest growth and yield: applications to mixed tropical forests. Wallingford, UK: CAB International.

Weibull, W. (1939). The phenomenon of rupture in solids. Proc. Royal Swedish Institute of Engineering Research (Ingeniorvetenskaps Akademiens Handlingar), 153, 1-55. 
Manuscrito recibido el 4 de septiembre de 2017

Aceptado el 23 de septiembre de 2019

Publicado el 13 de diciembre de 2019

\section{Este documento se debe citar como:}

Pérez-López, E., Santiago-García, W., Quiñonez-Barraza, G., Rodríguez-Ortiz, G., Santiago-García, E., \& Ruiz-Aquino, F. (2019). Estimación de distribuciones diamétricas para Pinus patula con la función Weibull. Madera y Bosques, 25(3), e2531626. doi: 10.21829/myb.2019.2531626
Madera y Bosques por Instituto de Ecología, A.C. se distribuye bajo una Licencia Creative Commons Atribución-NoComercialCompartirlgual 4.0 Internacional. 